\title{
LA HOLMQUISTITE DES PEGMATITES DE VOLTA GRANDE PRES DE SÃO JOÃO DEL REI, MINAS GERAIS, BRESIL: CARACTERISTIQUES CHIMIQUES ET MINERALOGIQUES
}

\author{
J. Quéméneur (*) \& M. Lagache (**)
}

\begin{abstract}
The Volta Grande pegmatites occur near the town of São João del Rei in the south of Minas Gerais state. They are associated with Early Proterozoic (Transamazonian) granites. These granites and pegmatites are hosted by gneisses and amphibolites of the Archean greenstone belt of the Rio das Mortes Valley. The pegmatites, very rich in $\mathrm{Li}$, develop a large metamorphic aureole in the amphibolites with holmquistite and zinnwaldite.

The holmquistite forms acicular or fibrous, purple coloured crystals. Individual crystals were analysed by electron-microprobe and wet chemistry. The structural formula $(\mathrm{Li}, \mathrm{Na})_{2}(\mathrm{Mg}, \mathrm{Fe})_{3}\left(\mathrm{Al}^{\mathrm{VI}}, \mathrm{Fe}\right)_{2}\left(\mathrm{Si}, \mathrm{Al}^{\mathrm{IV}}\right)_{8}$ $\mathrm{O}_{22}(\mathrm{OH}, \mathrm{F})$, is very similar to that proposed by London \& Burt (1982). The Volta Grande holmquistite is characterized by a particularly high $\mathrm{Fe} / \mathrm{Mg}$ ratio. A good correlation of $\mathrm{Fe} / \mathrm{Mg}$ ratios of the holmquistite and of the hornblende is observed in Volta Grande and Edison (Walker et al. 1989). We suggest that the holmquistite thas been formed by substitution of the hornblende of the wall-rock, caused by a Li-rich solution.

The unit cell parameters calculated with 25 peaks are comparable to those of Barraute (Nickel et al. 1960) and Greenbushes (Frost et al. 1987).
\end{abstract}

\section{RESUMO}

Os pegmatitos transamazônicos de Volta Grande ocorrem na região de São João del Rei, Minas Gerais, onde eles estão encaixados em anfibolitos do greenstone belt arqueano do vale do Rio das Mortes. Estes pegmatitos, muito ricos em lítio, desenvolvem uma auréola de metamorfismo de contato caracterizada pela formação de holmquistita e de Mg-zinnwaldita. A holmquistita aparece na forma de cristais fibrosos de cor violeta. A sua composição química foi determinada por microssonda eletrônica e química clássica, ela corresponde à fórmula $(\mathrm{Li}, \mathrm{Na})_{2}(\mathrm{Mg}, \mathrm{Fe})_{3}\left(\mathrm{Al}^{\mathrm{VI}}, \mathrm{Fe}\right)_{2}\left(\mathrm{Si}, \mathrm{Al}^{\mathrm{IV}}\right)_{8} \mathrm{O}_{22}(\mathrm{OH}, \mathrm{F})$, muito parecida com a fórmula proposta por London \& Burt (1982).

A holmquistita de Volta Grande é caracterizada por seu alto teor em Fe e o alto valor da relação Fe/Mg sendo assim parecida como a de Edison no Dakota do Sul (Walker et al. 1989). Geneticamente a holmquistita parece ter sido formada por substituição, às expensas da hornblenda, por ação de soluções oriundas dos pegmatitos. Pode-se notar a boa correlação entre as relações Fe/Mg das holmquistitas e das hornblendas encaixantes tanto em Volta Grande como em Edison. A presença simultânea de holmquistita e Mg-zinnwaldita sugere que as soluções eram ricas em $\mathrm{Li}, \mathrm{K}, \mathrm{Rb}$ e $\mathrm{H}_{2} \mathrm{O}$.

Os parâmetros de malha calculados sobre 25 picos estão em conformidade com os dados mais recentes tais como os de Greenbushes na Autralia (Frost et al. 1987).

\section{INTRODUCTION}

Il existe à Volta Grande près de São João del Rei, au sud de l'état de Minas Gerais (Fig. 1), un groupe de grandes pegmatites du type à albite et spodumène (Cerny 1990) caractérisées par leur richesse en $\mathrm{Li}$ $\left(1,5 \% \mathrm{Li}_{2} \mathrm{O}\right), \mathrm{Rb}\left(0,8 \% \mathrm{Rb}_{2} \mathrm{O}\right)$ (Quéméneur \& Lagache 1994 et Lagache \& Quéméneur, sous presse), Sn et Ta. Elles sont actuellement exploitées pour $\mathrm{Li}$, Sn et $\mathrm{Ta}$.

Ces pegmatites sont associées à un complexe granitique du Précambrien Inférieur ou Transamazonien (Fig. 2) daté, par la méthode $\mathrm{Rb} / \mathrm{Sr}$, de $1932 \pm 20$ Ma (Quéméneur \& Vidal 1989). Granites et pegmatites sont intrusifs dans un ensemble de type greenstone belt formé d'amphibolites, ultrabasites et gneiss d' âge probablement Archéen (Fig. 3).

Les pegmatites de Volta Grande forment de grandes lentilles allongées parallèlement à la foliation des amphibolites encaissantes (Fig. 4). Les dimensions de ces corps sont de l'ordre de 1000 x 100 x 20m (par exemple le corps A, Fig.4). Les amphibolites correspondent à des basaltes tholéitiques métamorphisés pendant l'Archéen Supérieur. Elles contiennent de fines intercalations d'origine sédimentaire riches en silicates de manganèse (spessartite) et graphite qui représentent d'anciens niveaux sédimentaires intercoulés.

Les pegmatites sont constituées par une bordure aplitique irrégulière d'albite microcristalline et d'une masse principale granitoide formée de spodumène (20$45 \%)$, quartz (30-40\%), albite (10-20\%), microcline (10-20\%) et de muscovite (2-10\%) selon Quéméneur (1987). Les pegmatites développent une importante auréole de métamorphisme de contact dont l'épaisseur peut atteindre $3 \mathrm{~m}$. Cette auréole commence par un liseré (0-1m) de glimmérite massive à zinnwaldite et, parfois, holmquistite au contact de la pegmatite. Puis vient une zone plus large (1-3m) d'amphibolite métamorphisée à holmquistite et zinnwaldite. L' amphibolite de cette dernière zone conserve sa trame originale avec les minéraux du métamorphisme régional, tels que hornblende, labrador et quartz, non transformés. 


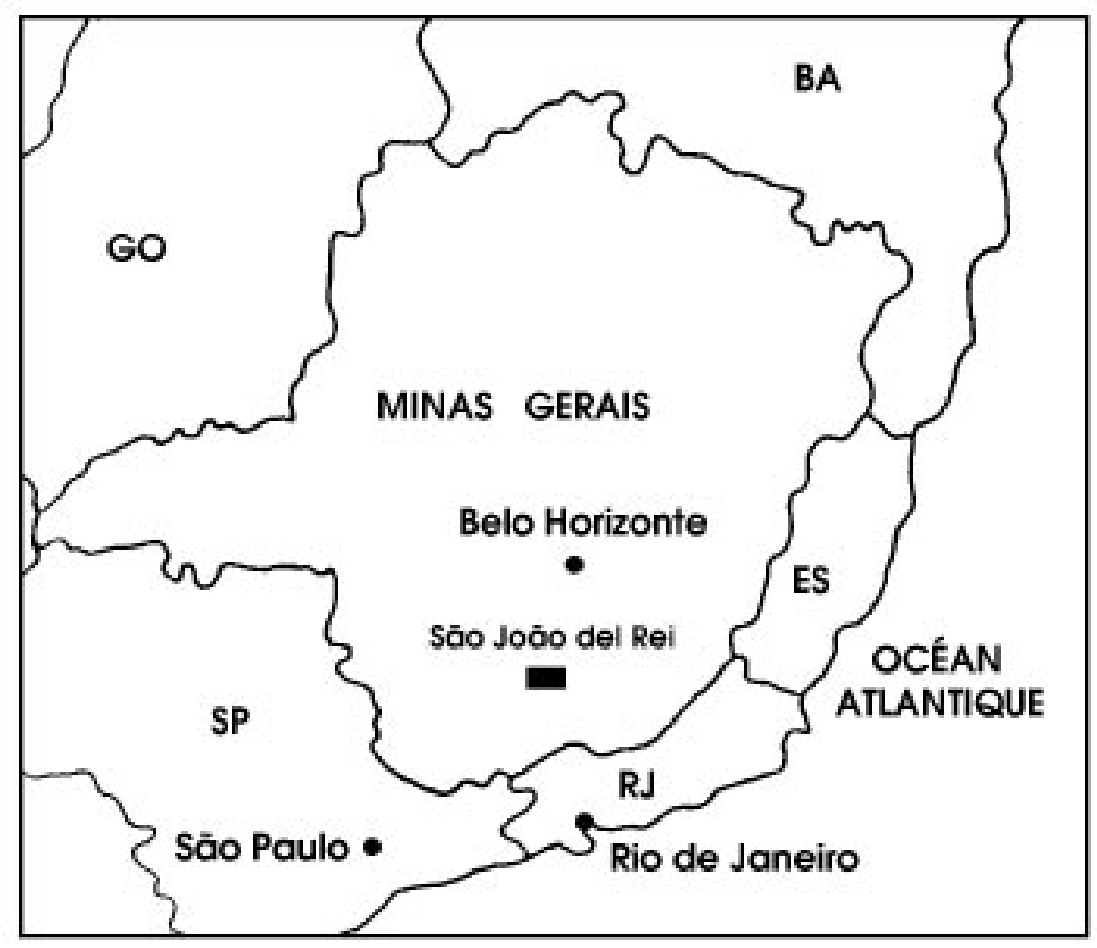

Figure 1: Localisation.

Figure 1: Localization sketch.

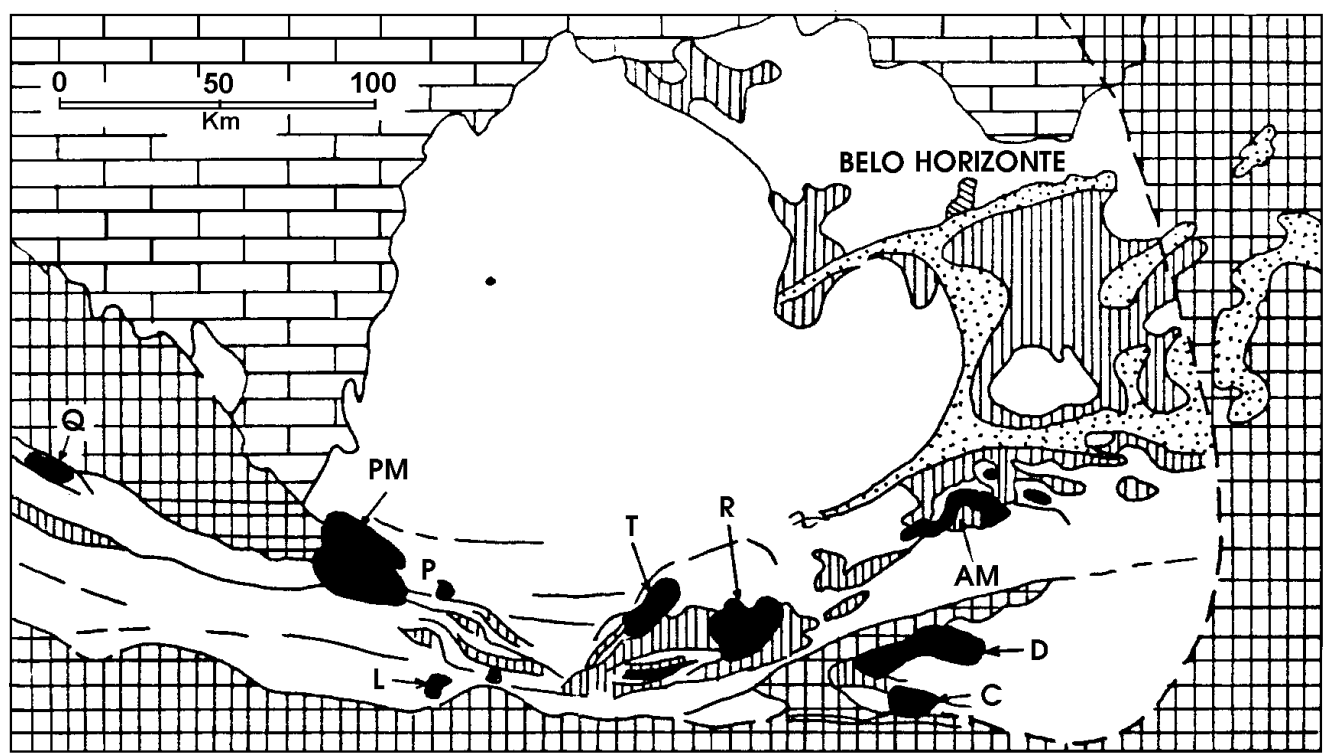

Granitoides transamazoniens

$\because$ Série méta sédimentaire du

$\therefore$ Super-groupe Minas

Gneiss, granites archéens
Greenstone belts archéens

Zone afectée par l'orogenèse brésilienne (Cambrien) Calcaires et schistes du Bambui (Précambrien Supérieur)

Figure 2: L'arc magmatique transamazonien du sud de Minas Gerais. Figure 2: The Transamazonic magmatic arc of southern Minas Gerais. 


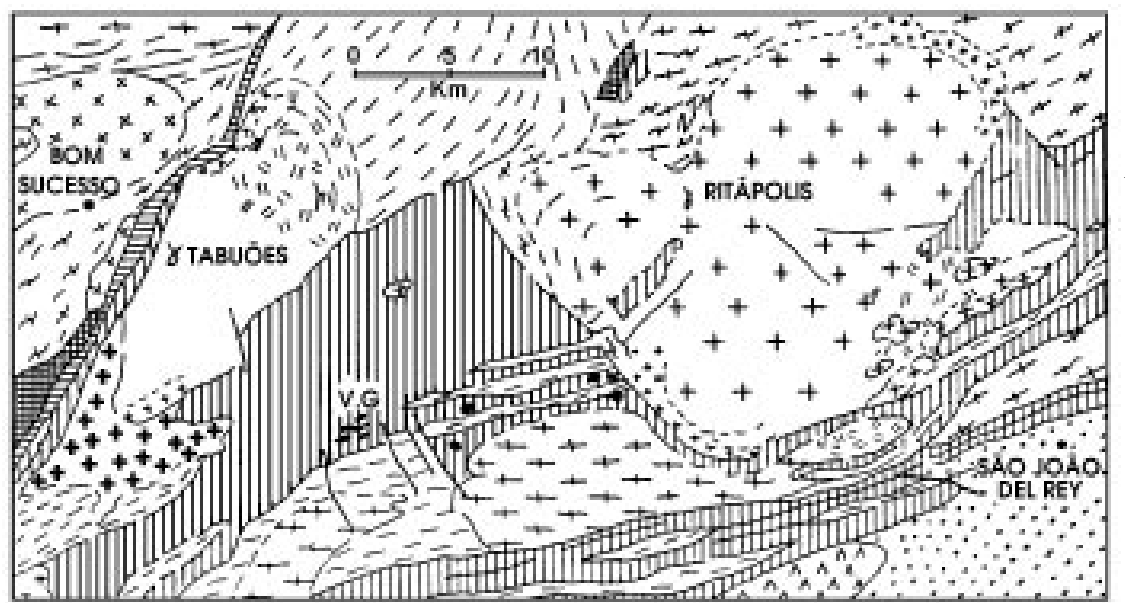

Figure 3: Géologie de la région de São João del Rei.

Figure 3: The geology of São João del Rei region.
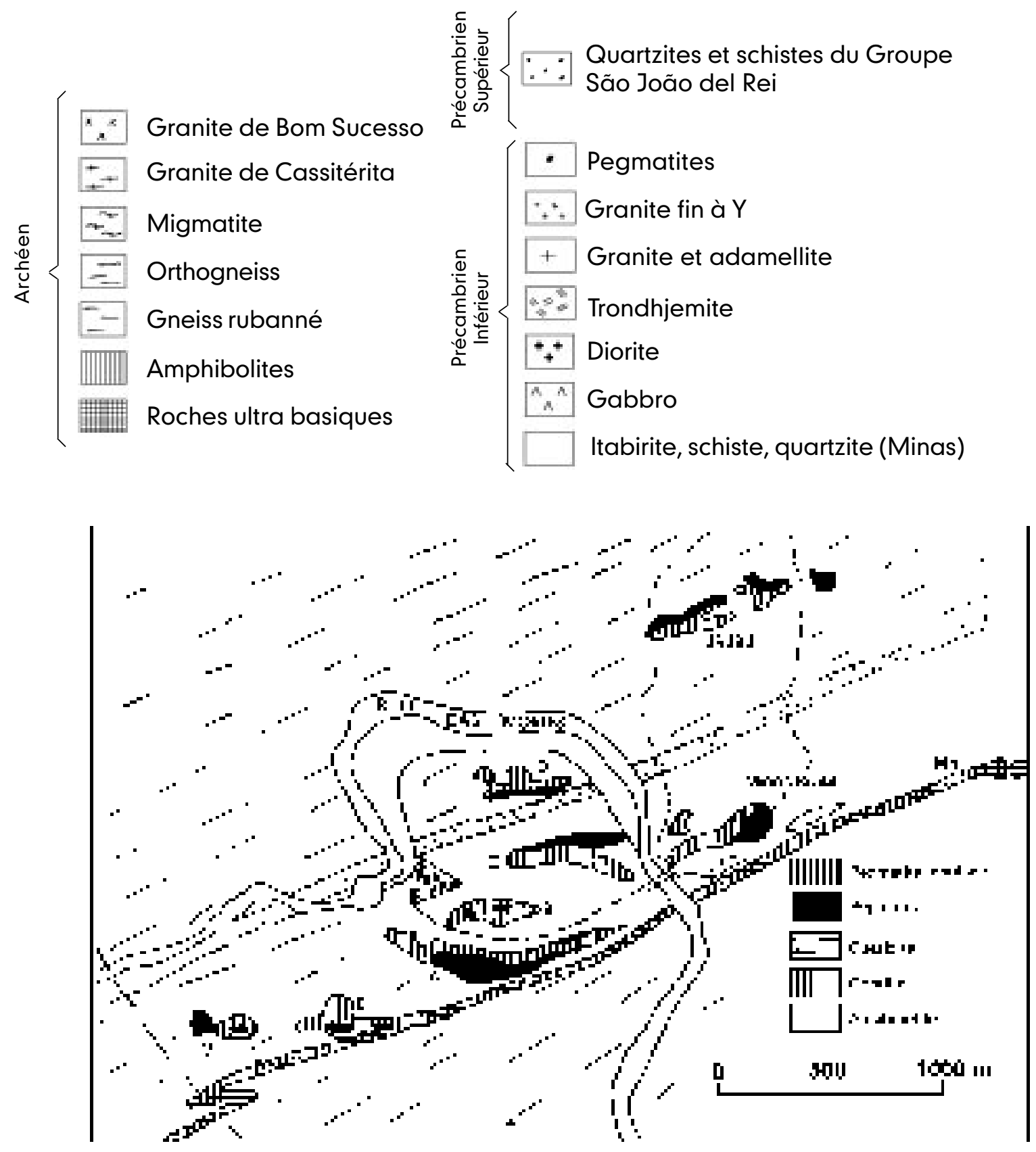

Figure 4: Les pegmatites de Volta Grande.

Figure 4: The Volta Grande pegmatites. 


\section{MORPHOLOGIE DE LA HOLMQUISTITE}

La holmquistite forme des cristaux de plusieurs $\mathrm{cm}$ de long, non orientés et surimposés à la texture de la roche; sa proportion varie de 10 à $30 \%$. Les cristaux aciculaires de section losangique ou lenticulaire ont une longueur qui varie de quelques $\mathrm{mm}$ à $30 \mathrm{~cm}$ pour un diamètre de 0,005 à $3 \mathrm{~mm}$. Ils présentent une couleur bleu-violet, particulièrement visible dans les quartz filonniens qui recoupent les amphibolites de l'auréole de contact. En effet, les cristaux de quartz de ces filons contiennent de très fines aiguilles de holmquistite (quelques mm de diamètre) leur donnant une teinte lilas et un aspect chatoyant (Karfunkel et al. 1994). Dans les zones pédogénétiquement altérées la holmquistite, minéral relativement résistant, forme une sorte de laine mauve.

\section{COMPOSITION CHIMIQUE}

Cinq échantillons provenant d'amphibolite à holmquistite et de glimmérite à zinnwaldite et holmquistite ont été analysés par microsonde électronique de type CAMEBAX à l'Université Paris 6 et au laboratoire de pétrographie de l'Université Clermont II. Un échantillon d' holmquistite a été analysé par voie chimique au laboratoire de géologie de l'Ecole Normale Supérieure. Les résultats de ces analyses sont rassemblés dans le tableau 1 .

Il apparait que la composition des différents échantillons analysés par microsonde électronique varie peu. Les plus fortes différences s'observent pour les teneurs en Fe, $\mathrm{Mg}$ et $\mathrm{Mn}$ mais la somme $\mathrm{Fe}+\mathrm{Mn}+\mathrm{Mg}$ exprimée en atomes reste pratiquement constante et est égale à 3 dans une formule à 24 oxygènes. La composition atomique moyenne de ces holmquistites, calculée à partir du tableau 1, est la suivante:

$\begin{array}{ll}\mathrm{Si} & 7,953 \\ \mathrm{Al} \text { IV } & 0,047 \\ \mathrm{Al} \mathrm{VI} & 1,993 \\ \mathrm{Fe} & 1,614 \\ \mathrm{Ti} & 0,066 \\ \mathrm{Mn} & 0,033 \\ \mathrm{Mg} & 1,392 \\ \mathrm{Na} & 0,105 \\ \mathrm{~F} & 0,060 \\ \mathrm{Li} & 1,8\end{array}$

Elle correspond donc à la formule théorique: $(\mathrm{Li}, \mathrm{Na})_{2}(\mathrm{Mg}, \mathrm{Fe}, \mathrm{Mn})_{3}\left(\mathrm{Al}^{\mathrm{VI}}, \mathrm{Fe}\right)_{2}\left(\mathrm{Si}, \mathrm{Al}^{\mathrm{IV}}\right)_{8} \mathrm{O}_{22}(\mathrm{OH}, \mathrm{F})_{2}$ telle qu'elle est proposée par London \& Burt (1982).

Les analyses obtenues dans ce travail sont également voisines de celles publiées dans la littérature (Tab. 2). On peut toutefois noter qu'elles sont plus basses pour le magnésium et corrélativement plus hautes pour le fer. Les holmquistites de Volta Grande paraissent, par ailleurs, être légèrement plus riches en sodium que la moyenne. Dans l'ensemble les holmquistites étudiées présentent une composition chimique voisine de celles de Tanco, Manitoba, Canada (Morgan \& London 1987), Edison, Black Hills, South Dakota,(Walker et al. 1989) et de celles de Green Bush en Autralie occidentale (Frost et al. 1987).

\begin{tabular}{|c|c|c|c|c|c|c|}
\hline Echant. & V154 & V158 & V158 & V159 & V183 & MB5(c \\
\hline $\mathrm{Si}_{2} \mathrm{O}$ & 59 & 59 & 58 & 57 , & 59 & 59 \\
\hline A & 12 & 12 & 12 & 12 & 12 & 12 \\
\hline F & & & & & & 3,66 \\
\hline F e 0 & 14 & 15 & 15 & 15 & 14 & 10 \\
\hline$M \cap O$ & 0,3 & 1 & 0,2 & 0,2 & 0,2 & $N . D$ \\
\hline $\mathrm{Mg} \mathrm{O}$ & 6,8 & 6 & 6,3 & 5,5 & 7 & 5,6 \\
\hline$C a O$ & 0 & 0 & 0 & 0 & 0 & 0,56 \\
\hline $\mathrm{No}_{2}$ & 0 & 0 & 0 & 0,2 & 0,3 & 0,2 \\
\hline 6 & 0 & 0,2 & 0 & 0 & 0 & 0,0 \\
\hline $\mathrm{T}_{2} \mathrm{O}$ & 0 & 0 & 0 & 0 & 0 & 0,1 \\
\hline Lबृ & 3,2 & 1 & 3,4 & 3,4 & 3 & 2,6 \\
\hline$F$ & & & 0,2 & & & $N . D$ \\
\hline Tot a & 96 & 97 & 96 & 95 & 97 & 94 \\
\hline
\end{tabular}

Tableau 1: Composition (poids \%) de holmquistites de Volta Grande, MG.

Table 1: Composition (in weight \%) of holmquistites from Volta Grande, Minas Gerais, Brazil. 


\begin{tabular}{|c|c|c|c|c|c|c|c|c|c|c|}
\hline & V.G. & Tanc & G.B. & Ed. & Azov & Bar. & Mt.Ma & Mt.Be & Kola & Utö \\
\hline $\mathrm{Si}_{2} \mathrm{O}$ & 58, & 55 & 59 & 59 & 58, & 59, & 59. & 59 & 58 & 60, \\
\hline$Q_{3}$ & 12 & 12 & 12 & 12 & 13 & 11 & 12, & 12 & 13 & 7 \\
\hline$F_{2}$ & 3,6 & & 1 & 4,4 & 1 & 2,9 & 1 & 2,3 & 2 , & 9,6 \\
\hline F e 0 & 11 & & 12 & 9,6 & 6,6 & 8 & 9,2 & $\begin{array}{ll}10 \\
\end{array}$ & 8 , & 4,3 \\
\hline $\mathrm{Fe}_{3}$ & 16 & 19 & 14 & 14 & 8,6 & 12 & 12 & 14 & 12 & $\begin{array}{ll}14 \\
\end{array}$ \\
\hline $\mathrm{MnO}$ & 0 & 0 & 0 & 0 , & 0 & 0 , & 0 & 0,2 & 0 , & \\
\hline $\mathrm{M} \mathrm{gO}$ & 6,4 & 6,6 & 8,3 & 7,6 & 11 & 10 & 10 & 8,8 & 9 & 12 \\
\hline $\mathrm{CaO}$ & 0 & 0 & 0 & 0 & 0 & 0,5 & 0 & 0,2 & 1 & \\
\hline $\mathrm{Na} \quad 2 \quad \mathrm{O}$ & 0 & 0 & 0 & 0 , & 0 & 0 & 0 & 0 & 0 & 1 , \\
\hline $\begin{array}{lll} & 2 & 0 \\
\end{array}$ & 0 & 0 & 0 & 0 & 0 & 0 , & 0,3 & 0 & 0 & 0,5 \\
\hline $\begin{array}{lll}\text { Li } & 2 & 0 \\
\end{array}$ & 2,6 & & 3 & 2,9 & 3,5 & 3,5 & 3,7 & 3,3 & 2 & 2 \\
\hline$F$ & 0 & 0 & 0 & & 0 & 0 & 0 & 0 & 0 , & 0 \\
\hline $\mathrm{OH}$ & 2,4 & & 1 & & 2,4 & 2 , & 2,0 & 2 & 1 & 2,5 \\
\hline
\end{tabular}

Tableau 2: Composition (poids \%) de quelques holmquistites.

Table 2: Composition (in weight \%) of some holmquistites.

V.G. = Volta Grande, $($ this paper) - Tanco, Manitoba, Canada (Morgan \& London 1987) - G.B.= Greenbushes, Australia, (Frost et al. 1987). - Ed=Edison pegmatite, South Dakota, (Walker et al. 1989). - Azov, Ukrainia, (Stolyarova, 1989).- Bar.= Barraute, Québec, (Nickel et al. 1960). - Mt Ma= Mt. Marion, Australia, (Willkins et al. 1970). - Mt Be= Mt. Benson, Zimbabwe, (Knorring \& Hornung 1961). Kola, Russia, (Ginsburg 1960). Utö, Sweden, (Osann 1913 et Palache et al. 1930).

\section{PARAMÈTRES DE MAILLE}

Les valeurs ont été calculées sur 25 pics en utilisant le diagramme $\mathrm{Cu} \mathrm{Ka}$; on obtient les résultats suivants (en $\AA$ ):

$$
\begin{aligned}
\mathrm{a} & =18,284 \pm 0,020 \AA \\
\mathrm{b} & =17,691 \pm 0,020 \AA \\
\mathrm{c} & =5,276 \pm 0,002 \AA \\
\mathrm{V} & =1706,725 \AA^{3}
\end{aligned}
$$

\begin{tabular}{|c|c|c|cc|c|}
\hline V.G. & G.B. & Bar. & Utö & Kola & Wein. \\
a 18,284 & 8,282 & 18,3 & 18,36 & 18,27 & 18,26 \\
b $17,69117,695 \$ 17,69$ & 17,75 & 17,71 & 17,679 \\
\hline C 5,2763 & 5,2705 & 5,3 & 5,29 & 5,3 & 5,2753 \\
\hline
\end{tabular}

Tableau 3: Paramètres de maille de quelques holmquistites en $\AA$.

Table 3: Lattice parameters of some holmquistites.

V.G., G.B., Bar., Utö, Kola: idem tableau 2,

Wein = Weinebene, Autriche (Göd 1978).

\section{CONCLUSION}

Bien que relativement rare, la holmquistite a été rencontrée dans les pegmatites du monde entier (London 1986):

- En Europe avec les gisements de Utö en Suède
(Osann 1913), de la péninsule de Kola (Ginsburg 1960), d'Azov (Bayrakov 1968) et de Weinebene en Carenthie (Walter \& Walitzi 1985);

- En Asie avec les pegmatites de l'Altaï (Zang Ru Bo et al. 1986) et de Tarynnak dans le bouclier de l'Aldan en Sibérie Orientale (Gorelov et al. 1983).

- En Afrique avec les gisements de Kirengo au Ruanda (Van Wambeke 1970), de Mt. Benson au Zimbabwe (Knorring \& Hornung 1961) et celui de Morrua au Mozambique (Barros 1972).

- En Australie occidentale avec les gisements de Mt. Marion (Willkins et al. 1970) et de Greenbushes (Frost et al. 1987).

- En Amérique du Nord on peut citer, entre autres, les pegmatites de Barraute au Québec (Karpoff 1960), celle de Tanco au Manitoba (Morgan \& London 1987), celle de Edison au Dakota du Sud (Shearer \& Papike, 1988; Walker et al. 1989).

- En Amérique du Sud les pegmatites de Volta Grande (présent travail).

Il apparaît qu' à de rares exceptions près (par exemple les pegmatites de l'Altaï encaissées dans des quartzites ferrifères à amphiboles), toutes ces pegmatites sont encaissées dans des roches basiques ou ultrabasiques de l'Archéen ou du Protérozoique Inférieur. Il semble qu'en second lieu la holmquistite ne se développe qu'à partir d' autres amphiboles : hornblende, actinolite ou trémolite; on ne cite pas d'exemple de holmquistite formée aux dépens de 
pyroxènes. La composition des holmquistites reflète, en général, celle des amphiboles substituées. Ainsi, dans la région d'Azov, la holmquistite, qui s'est développée au sein d'une roche à trémolite-actinolite, présente t-elle un rapport $\mathrm{Fe} / \mathrm{Mg}$ bas $(0,383)$; alors que celles de Edison et surtout de Volta Grande et de Tanco ont des rapports $\mathrm{Fe} / \mathrm{Mg}$ beaucoup élevés (respectivement 0,93-1,08; 2,37-3,51 et 3,22-3,54) car elles se forment aux dépens de hornblendes ferrifères (tab.4). Des analyses détaillées montrent à Edison et Volta Grande, une excellente corrélation dans chaque échantillon, entre les valeurs des rapports $\mathrm{Fe} / \mathrm{Mg}$ des holmquistites et des hornblendes.

Ces résultats confirment l'hypothèse selon laquelle la holmquistite se forme par substitution des amphiboles des roches encaissantes sous l'action de solutions hydrothermales provenant d'un "magma" pegmatitique riche en lithium. Un tel magma est aussi, en général, riche en $\mathrm{K}$ et $\mathrm{Rb}$, ce qui explique l'association très fréquente de l'holmquistite avec la zinnwaldite ou la biotite lithinifère; ces micas présentent souvent de fortes teneurs en $\mathrm{Rb}$ et $\mathrm{Cs}$ comme, par exemple à Volta Grande (Quéméneur \& Lagache 1994; Lagache \& Quéméneur, sous presse) et à Urubu au NE de l'état de Minas Gerais (Quéméneur et al. 1993).

\section{REMERCIEMENTS}

Ce travail a été réalisé grâce à l'appui financier et analytique du projet CAPES-COFECUB 87/81 et de la FAPEMIG. Nous tenons aussi à remercier la direction de la MIBRA pour les facilités accordées sur le terrain.

\begin{tabular}{|c|c|l|l|l|c|c|c|}
\hline Fe/Mg & Tanc & V159 & V15 & V18 & Ed.1 & Ed.2 & Ed.3 \\
\hline H $\circ$ r b $\mid$ e n & 3,2 & 3,3 & 3, & 2, & 1 & 1 & 1 \\
\hline H $\circ$ । m q u i s & 3,5 & 3,5 & 2, & 2, & 0, & 1 & 1 \\
\hline
\end{tabular}

Tableau 4 : Rapports Fe/Mg des hornblendes et des holmquistites de Tanco, Volta Grande et de Edison (Ed.1, 2 et 3)

Table 4: Fe/Mg ratios of hornblendes and holmquistites from Tanco; Volta Grande, Minas Gerais, Brazil (V159, 158, 183) and Edison, Black Hills, USA (Ed.1, 2, 3).

\section{REFERENCES BIBLIOGRAPHIQUES}

BARROS, R.F. de 1972. Nota sobre uma ocorrência de holmquistite na bordadura do pegmatito Morrua (Zambezia), Mozambique. Rev. Univ., Fac Ciênc. Lisboa, Ser. 26 (Ciênc. Nat.). 17: 175186.

BAYRAKOV, V.V. 1968. Kholmkvistite-asbest iz Priazov'ya (holmquistite-asbeste de la région d'Azov). Dock. Akad. Nauk. USSR.. 178: 420-423.

CERNY, P. 1990. Distribution, affiliation and derivation of rareelement granitic pegmatites in the Canadian Shield. Geol. Rundschau. 79: 183-226.

FROST, M.T.; TAMBOURAKIS, G. \& DAVIS, J. 1987. Holmquistite-bearing amphibolite from Greenbushes, western Australia. Mineral. Mag. 51: 585-591.

GINSBURG, I.V.; ROGACHEV, D.L. \& BONDAREVA, A.M. 1958. New data on holmquistite. Dokl. Akad. Nauk USSR.. 119: 10131016.

GÖD, R. 1978. Vorläufige Mitteilung über einen SpodumenHolmquistite führenden Pegmatit aus Kärnten. Österreich. Akad. Wiss. Math.-Natur. Wiss. K1. Anz. 115: 161-165.

GORELOV, G.F.; GORB, A.M.; KOSUKHINA, I.G.; SAFONOF, A.F. \& SHESTOKOV, A.V. 1983. A holmquistite from the Tarynnak ore deposit of ferruginous quartzites in the Chara-Toko area of the Aldan Shield. Soviet Geol. \& Geophys. 24: 129-131.

KARFUNKEL, J.; QUEMENEUR, J. \& WEGENER, R. 1994. Quartz-holmquistite cat's eye from Brazil. Can. Gemmologist. (sous presse).

KARPOFF, B. 1960, Holmquistite occurrences in the mining property of Québec Lithium Corporation, Barraute, Québec. In: INTERN. GEOL. CONG., 21. Copenhagen, 1960. Rept. 17: 7-14.

KNORRING ,O. Von \& HORNUNG, G. 1961. On the lithium amphibole holmquistite, from Benson mine, Mokto, Southern Rhodesia. Mineral. Mag. 32: 731-735.

LAGACHE, M. \& QUEMENEUR, J. (sous presse). The Volta Grande pegmatites (Minas Gerais, Brazil): an example of rareelement pegmatites exceptionally enriched in lithium and rubidium. Can. Mineral.

LITVIN, M.A. \& PATRONOV, A.N. 1984. The use of the optical absorption spectra for the refinement of $\mathrm{Fe}(+3)$ ions distribution in the holmquistite structure. Mineralogischeskiy -Sbornik(L'vov). 38: 27-32.
LONDON, D. \& BURT, D.M. 1982. Lithium minerals in pegmatites. MAC short course handbook. 8: 93-133.

LONDON, D. 1986. Holmquistite as guide to pegmatite rare metal deposits. Economic Geology. 81: 704-712.

MORGAN, G. \& LONDON, D. 1987. Alteration of amphibolitic wallrocks around the Tanco rare-element pegmatite, Bernic Lake, Manitoba. Amer. Mineral. 72: 1097-1121.

NICKEL, E.H.; MAXWELL, J.H. \& ROWLAND, J.F. 1960. Holmquistite from Barraute, Québec. Can. Mineral. 6: 504-512.

OSANN, A. 1913. Über Holmquistit, einen Lithiumglaukophan von der Insel Utö. Sitzungber. Heidelberger Akad. Abh. A. 23: 116.

PALACHE, C.; DAVIDSON, S.C. \& GORANSON, E.A. 1930. The Hiddenite deposit in Alexander County, North Carolina. Am. Mineral. 15: 280-302.

QUEMENEUR, J. 1987. Petrography of the pegmatites from the Rio das Mortes Valley, southeast Minas Gerais, Brasil.Proc. ISGAM. Rev. Bras. Geoc. 17: 595-600.

QUEMENEUR, J.; LAGACHE, M. \& CORREIA NEVES, J. M. 1993. La pegmatite Urubu, Araçuai, Minas Gerais (Brésil), exemple de pegmatite complexe à pétalite: zonalité minéralogique et géochimie des micas et des tourmalines. C.R. Acad. Sci. Paris. 317, II: 1425-1431.

QUEMENEUR, J. \& LAGACHE, M. 1994. The Volta Grande pegmatites (Minas Gerais, Brazil): granitic pegmatites rich in $\mathrm{Rb}$ and rare elements. GENERAL MEETING, INTERN. MINERAL. ASSOC.,16. Proceedings.... 342.

QUEMENEUR, J. \& VIDAL, P. 1989. Primeira datacão radiometrica dos granitos da região de São João del Rei (MG). In: SIMP. GEOL. MINAS GERAIS, 4. Belo Horizonte-MG, 1989.Anais... 7, 135-148.

SHEARER, C.K. \& PAPIKE, J.J. 1988. Holmquistite-bearing amphibolite, Edison Pegmatite, Black Hills, South Dakota. Amer. Miner. 73: 324-337.

STOLYAROVA, T.A.; PORITSKAYA, L.G. \& KOTOV, N.V. 1989. Thermodynamic study of holmquistite. Geokhimiya . 2: 313318.

VOGT, T.; BASTIANSEN, O. \& SKANCKE, P. 1958. Holmquistite as rhombic amphibole. Amer. Mineral. 43: 981-982.

WALTER, F. \& WALITZI, E.M. 1985. Holmquistite von Brandrücken (Weinebene) Koralpe/Kärnten; ein Vorbericht. Landesmus. Abt. Mineral. A-8010 Gratz. Die Karinthin. 92: 245-248. 
WALKER, R.J.; HANSON, G.N. \& PAPIKE, J.J. 1989. Trace element constraints on pegmatites genesis; Tin Mountain Pegmatite, Black Hills, South Dakota. Contrib. Mineral. Petrol. 101: 290300.

WAMBEKE, (Van) L. 1970. Contribution à l'étude de l'holmquistite bleue de Kirengo, république du Ruanda. Ruanda Serv. Geol. Bull. 6: 15-23.
WILLKINS, R.W.T.; DAVIDSON, L.R. \& ROSS, J.R. 1970. Occurrence and infrared spectra of holmquistite and hornblende from Mt. Marion, near Kalgoorlie, western Australia. Beitrag Mineral. Petrol. 28: 280-287.

ZANG RU BO. 1986. The lithium minerals of China's Li pegmatites. In: GENERAL MEETING OF THE IMA., 14. Stanford, 1986. Proceedings..., CA. p. 280 . 
